Solid State Communications, Vol. 36, pp. 249-251.

Pergamon Press Ltd. 1980. Printed in Great Britain.

\title{
LONGITUDINAL SPIN POLARIZATION AND SYMMETRIES IN LOW-ENERGY-ELECTRON DIFFRACTION: EXPERIMENT AND THEORY FOR Pt (111)
}

\author{
P. Bauer* \\ Max-Planck-Institut für Plasmaphysik, Euratom-Association, SFB 128, D-8046 Garching bei München, Germany \\ R. Feder \\ Theoretische Festkörperphysik, FB 10, Universität Duisburg GH, D4100 Duisburg, Germany \\ and \\ N. Müller
}

Fachbereich 4, Festkörper und Festkörpertechnologie, Universität Osnabrück, D-4500 Osnabrück, Germany

(Received 6 June 1980 by P.H. Dederichs)

\begin{abstract}
In low-energy electron diffraction from $\mathrm{Pt}(111)$, the longitudinal component of the spin polarization vector and its transverse component normal to the scattering plane were measured by a Mott detector and found to agree very well with corresponding theoretical results. Rotation diagrams of the longitudinal and transverse components exhibit only a three-fold symmetry in contrast to the six-fold symmetry, which time reversal invariance dictates for intensities.
\end{abstract}

IN ELASTIC electron-atom scattering with an unpolarized incident beam of momentum $k_{0}$, spin-orbit coupling leads to spin polarization such that for electrons of momentum $\mathbf{k}$ the polarization vector is normal to the scattering plane defined by $k_{0}$ and $k$ [1]. In low-energy electron diffraction from crystal surfaces of large- $Z$ materials effects of spin polarization normal to the scattering plane are well established experimentally and theoretically [2]. So far, however, no experimental verification is known for in-plane components, the existence of which was predicted by theory [3] as a consequence of multiple scattering. We report here, for diffraction from $\mathrm{Pt}(111)$, the first measurements of a spin polarization component parallel to the scattering plane and compare them to corresponding theoretical results. The present spin polarization versus azimuthal angle profiles (rotation diagrams) further clarify a lack of rotation symmetry indicated by earlier results on the normal component of polarization, which were measured for a slightly different diffraction geometry [4], and provide experimental verification of recent predictions based on symmetry arguments [5].

Most of the experimental set-up has been described previously [4]. The principle of measuring the longitudinal spin polarization is illustrated with the aid of Fig. 1. The moveable electron gun and the

\footnotetext{
* Present address: Universität Linz, Institut für Experimentalphysik, A-4045 Linz-Auhof, Austria.
}

high-precision two-axis manipulator for the $\mathrm{Pt}(111)$ crystal allow to feed selected diffracted beams into the polarization analyzer based on Mott scattering at $120 \mathrm{keV}$ [1]. On its path between the diffraction system and the Mott-detector, the diffracted beam under consideration is deflected electrostatically by $90^{\circ}$ about the axis normal to the scattering plane $\left(k_{0}, k\right)$. Since the deflection leaves the polarization vector $P$ unchanged, the transverse component $P_{n}$ normal to the scattering plane $\left(P_{n}=\mathbf{P} \cdot \mathbf{n} ; \mathbf{n}=\mathbf{k}_{0} \times \mathbf{k} /\left|\mathbf{k}_{0} \times \mathbf{k}\right|\right)$ remains transverse with respect to the new beam direction, while the initial longitudinal component $P_{k}=\mathrm{P} \cdot \mathrm{k} /|\mathrm{k}|$ becomes transverse (lying in the paper plane, still normal to $n$ ). The scattering chamber of the Mott detector can be rotated about the incoming beam. Setting the scattering plane of the Mott detector parallel to the original scattering plane $\left(k_{0}, k\right)$ one measures $P_{n}$. The component $P_{k}$ is analysed, if the two scattering planes are perpendicular to each other.

Numerical calculations were performed according to a relativistic LEED theory [6] with model assump. tions as in a recent application to $\operatorname{Pt}(111)$ [4]. The effective ion-core potential contains an energydependent local exchange contribution, the inner potential has the real and imaginary parts 12 and $4 \mathrm{eV}$, and the surface barrier is exponentially smooth. In addition to the normal component $\boldsymbol{P}_{\boldsymbol{n}}$ and the longitudinal component $P_{k}$, we have also calculated the second inplane component $P_{\mathrm{e}}=\mathrm{P} \cdot \mathrm{e}$, where e $=\mathrm{k} \times \mathrm{n} / k$.

Figure 2 shows typical calculated diagrams for the 


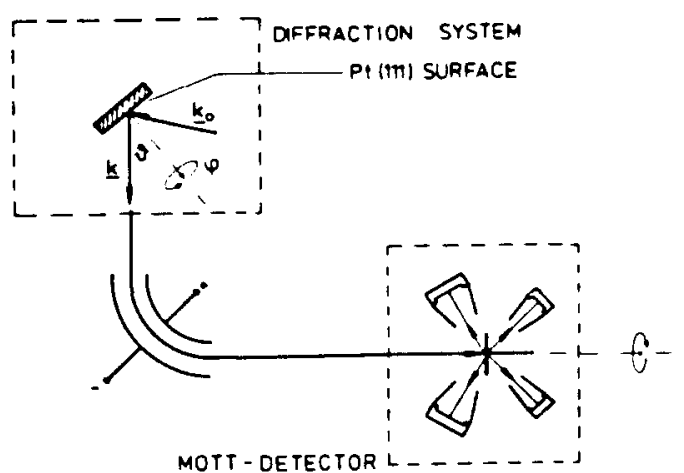

Fig. 1. Schematic experimental set-up.

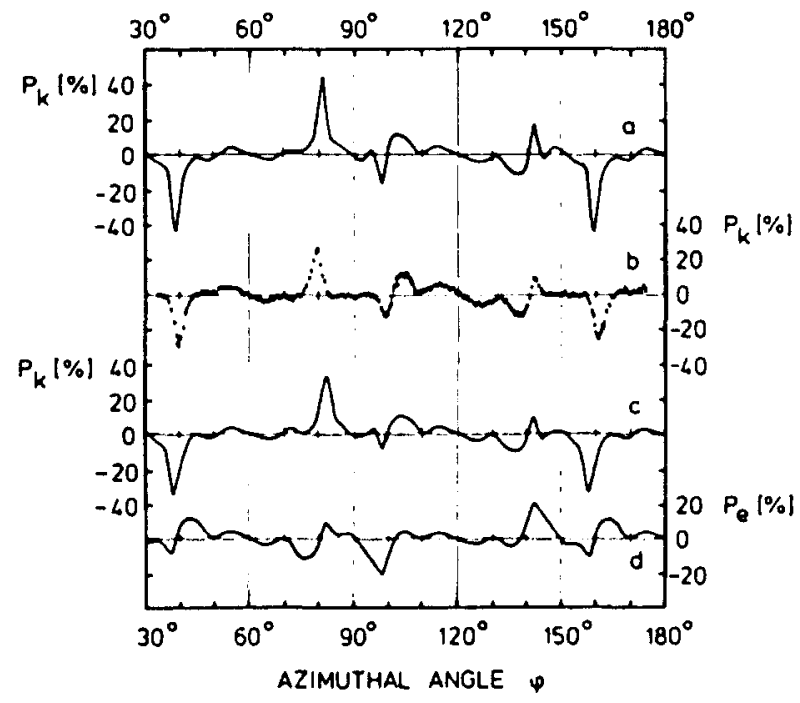

Fig. 2. Rotation diagrams for the specular beam polarization components lying in the scattering plane. (a) Calculated longitudinal component $P_{k}(\varphi)$ for polar angle $\theta=43^{\circ}$, and electron energy $E=60 \mathrm{eV}$. (b) Measured $P_{k}(\varphi)$ for $\theta=43.5^{\circ} \pm 0.5^{\circ}$ and $E=60 \pm 1 \mathrm{eV}$. The error bars give the statistical error due to counting in the Mott detector. Additional experimental errors are: Polarization zero $\pm 1 \%$, calibration of polarization $\pm 7 \%$ of given values, linearity of azimuthal angle $\pm 1^{\circ}$. (c) Calculated $P_{k}(\varphi)$ for $\theta=44^{\circ}$ and $E=60 \mathrm{eV}$. (d) Calculated in-plane component $P_{e}(\varphi)$ (perpendicular to $k$ and $n$ ) for $\theta=44^{\circ}$ and $E=60 \mathrm{eV}$.

components $P_{k}$ and $P_{e}$ of the polarization vector and the measured diagram for the longitudinal component $\boldsymbol{P}_{\boldsymbol{k}}$. For the latter we note firstly the sizeable peak values (up to $30 \%$ ), secondly the strong variations over small angular ranges and thirdly the agreement between experiment and theory. Similar properties are found for the corresponding normal spin polarization $\boldsymbol{P}_{n}$, which is given in Fig. 3. We notice correlations between the components: at angles at which $P_{n}$ shows sharp peaks, also $P_{k}$ and $P_{e}$ have peaks or double-peak structures.

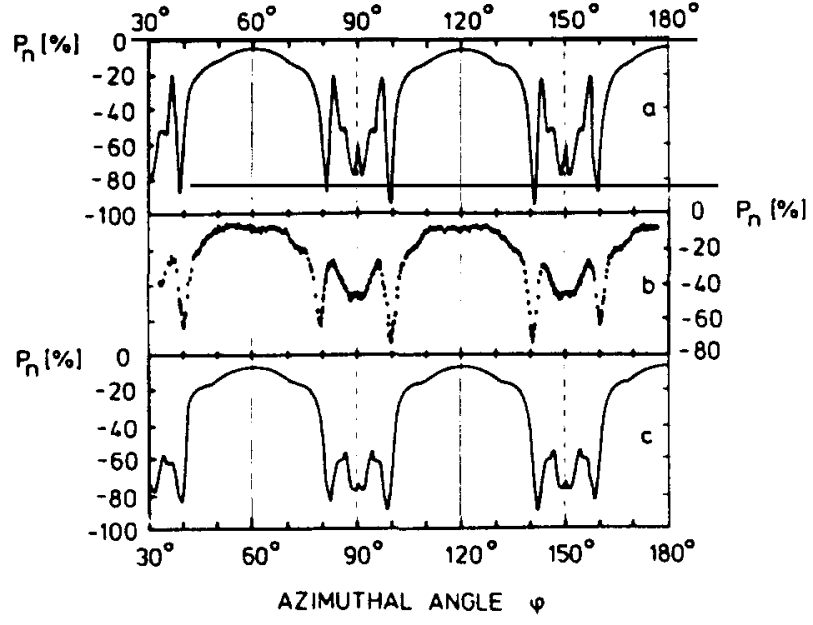

Fig. 3. Rotation diagrams for the polarization component normal to the scattering plane. (a) Calculated $P_{n}(\varphi)$ for $\theta=43^{\circ}$ and $E=60 \mathrm{eV}$. (b) Measured $P_{n}(\varphi)$ for $\theta=43.5^{\circ} \pm 0.5^{\circ}$ and $E=60 \pm 1 \mathrm{eV}$ [uncertainties see Fig. 2(b)]. (c) Calculated $P_{n}(\varphi)$ for $\theta=44^{\circ}$ and $E=60$ $\mathrm{eV}$.

Turning to the symmetry properties of the rotation diagrams in Figs. 2 and 3 we notice that $P_{k}$ and $P_{e}$ vanish for $\varphi=60^{\circ}$ and $120^{\circ}$, i.e. $P$ is normal to the scattering plane if the scattering plane is a mirror plane of the crystal. While $P_{n}$ is symmetric with respect to the mirror planes, $P_{k}$ and $P_{e}$ are antisymmetric. This behaviour is a consequence of the fact that $P$, which is essentially an angular momentum, is an axial vector, i.e. it can be thought of as the vector product of two polar vectors. Since the existence of mirror planes separated by $60^{\circ}$ angles implies three-fold rotation symmetry about the surface normal, we conclude that our rotation diagrams show the three-fold symmetry of the crystal. That the symmetry is in fact not a higher one, is apparent from the discrepancy between the heights of the peaks of $P_{n}$ near $\varphi=80^{\circ}$ and $\varphi=100^{\circ}$ and the behaviour of $P_{k}$ in a wide angular range around $\varphi=80^{\circ}$ and $\varphi=100^{\circ}$. Also there is no mirror or antimirror symmetry with respect to $\varphi=90^{\circ}$ and $\varphi=150^{\circ}$, which should be present if the rotation symmetry were sixfold.

Our results contain very interesting information on time reversal effects. In analogy to the behaviour of LEED intensity rotation diagrams, which due to the reciprocity theorem have higher symmetry than the crystal [7], one could naively expect six-fold rotation symmetry also for the polarization vector $\mathbf{P}$. Our finding of only three-fold rotation symmetry is, however, consistent with time reversal symmetry, which entails, for the specular beam from a surface with three-fold symmetry, six-fold symmetry only for the total length $P$ of the polarization vector (the degree of polarization), but not for its individual components [5]. 
In conclusion, we have demonstrated by experiment and numerical calculation that in spin-polarized lowenergy electron diffraction (SPLEED) the polarization vector $\mathbf{P}$ of the specular beam has in general nonvanishing components lying in the scattering plane, which obey the spatial symmetry given by the crystal. Especially, time reversal symmetry does not lead to an increase of the symmetry of $P$ beyond the symmetry inherent in the geometry of the diffraction set-up.

Acknowledgements - We would like to thank Dr W. Eckstein and Prof. Dr D. Menzel for their support of the experimental part of this work. Our gratitude extends to Prof. Dr G. Eilenberger for encouragement and the kind hospitality of the Institut für Festkörperforschung of the KFA Jülich.

\section{REFERENCES}

1. J. Kessler, Polarized Electrons. Springer, Berlin (1976).

2. Review article by R. Feder, [J. Phys. C (1980), - to be published] and references therein.

3. R. Feder, Surf. Sci. 63, 283 (1977).

4. P. Bauer, R. Feder \& N. Muiller, Surf. Sci 99, L395 (1980).

5. R. Feder, Phys. Lett. 78A, 103 (1980).

6. R. Feder, Phys. Status Solidi (b) 49 (1972) 699; Phys. Rev. Lett. 36, 598 (1976).

7. D.P. Woodruff \& B.W. Holland, Phys. Rett. 31A, 207 (1970). H.E. Farnsworth, Phys. Lett. 36A, 56 (1971); M.G. Lagally, T.C. Ngoc \& M.B. Webb, Surf. Sci. 25, 444 (1971). 